\title{
Gradhiva
}

GRADHIV

Revue d'anthropologie et d'histoire des arts

16 | 2012

Chines, l'État au musée

\section{Nicolas Puig. Farah. Musiciens de noces et scènes}

urbaines au Caire

Arles, Actes Sud-Sindbad, coll. « La Bibliothèque arabe », 2010, 218 p.

Jean-Charles Depaule

\section{OpenEdition}

Journals

Édition électronique

URL : http://journals.openedition.org/gradhiva/2534

DOI : 10.4000/gradhiva.2534

ISSN : 1760-849X

Éditeur

Musée du quai Branly Jacques Chirac

Édition imprimée

Date de publication : 1 décembre 2012

Pagination : 254-255

ISBN : 978-2-35744-048-7

ISSN : 0764-8928

Référence électronique

Jean-Charles Depaule, « Nicolas Puig. Farah. Musiciens de noces et scènes urbaines au Caire », Gradhiva [En ligne], 16 | 2012, mis en ligne le 13 décembre 2012, consulté le 21 septembre 2020. URL : http:// journals.openedition.org/gradhiva/2534; DOI : https://doi.org/10.4000/gradhiva.2534

Ce document a été généré automatiquement le 21 septembre 2020.

(c) musée du quai Branly 


\section{Nicolas Puig. Farah. Musiciens de noces et scènes urbaines au Caire}

Arles, Actes Sud-Sindbad, coll. « La Bibliothèque arabe », 2010, 218 p.

Jean-Charles Depaule

\section{RÉFÉRENCE}

Nicolas Puig. Farah. Musiciens de noces et scènes urbaines au Caire. Arles, Actes SudSindbad, coll. « La Bibliothèque arabe », 2010, 218 p.

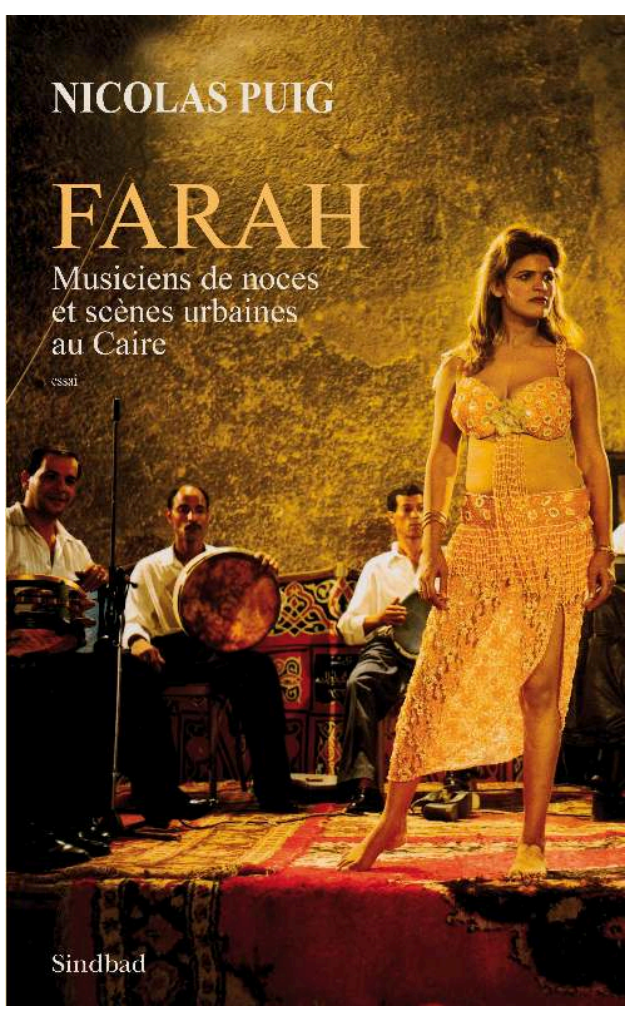


1 Au Caire, la nuit venue, au détour d'une rue, dans un quartier populaire ancien ou moderne, il arrive que l'attention du promeneur soit attirée par un lieu où se concentrent une vive lumière, une musique et des voix très amplifiées: devant un public d'hommes et de femmes, assis séparément, des musiciens installés sur un podium encadré de tentures multicolores accompagnent un chanteur ou une chanteuse, et une danseuse très maquillée au corps en partie dénudé. Un homme vocifère, des billets à la main. Des enfants s'amusent, courent.

2 C'est ce qu'on nomme farah. En arabe, le mot signifie « joie », et par extension il désigne dans le parler égyptien des festivités, et les noces en particulier. Nicolas Puig a mené une enquête auprès des « musiciens dont la rue est la scène ", ces artistes qui, au Caire, sont inséparables des fêtes populaires baladi («authentiques, locales ", par opposition à afrangi, «à l'occidentale»). Mais l'univers musical et urbain dont ils sont les représentants semble voué à la disparition et le livre s'achève sur une note désenchantée, même si les Cairotes «ne peuvent se départir d'une sorte d'affection » à leur égard. Le lecteur était prévenu: dès les premières lignes l'auteur citait cette remarque d'un de ses interlocuteurs, qui vaut pour les destinées aussi bien individuelles que collectives: «La vie du musicien est comme la vapeur d'eau, elle monte et disparaît. »

3 La première partie de ce bel ouvrage restitue la richesse de la matière ethnographique recueillie par Nicolas Puig, où les enregistrements audiovisuels occupent une place significative - il parle à ce propos de "carnets vidéo». Il y explore en outre la profondeur historique d'une tradition musicale populaire dont l'évolution est indissociable des recompositions des territoires de la ville ${ }^{1}$. La seconde partie est composée de quatre récits de musiciens : celui d'un multi-instrumentiste (à qui le livre est dédié), considéré comme un maître, et ceux de sa fille et de deux percussionnistes.

Outre les musiciens, chanteur(s) et instrumentistes (au minimum un percussionniste et un joueur d'instrument mélodique - généralement un clavier électronique, urg), et la danseuse, l'acteur indispensable d'un farah est l'«ambiancier ». À la différence de l'organisateur, chargé de s'entremettre entre commanditaire et musiciens, qui est lui peu visible, l'ambiancier, nabatshi (littéralement "préposé »), se distingue par ses incessantes et très sonores interventions et par le fait qu'il est constamment en mouvement, agitant une liasse de billets de banque qu'il fait parfois pleuvoir sur le public. Il salue les arrivants en déclinant leurs qualités et en les localisant dans la géographie du Caire, recueille leurs dons en espèces, qui seront répartis entre les artistes et les mariés, et célèbre leur générosité. Ses propos, soulignés par une phrase mélodique, participent à l'« esthétique de la saturation » qui, pour Nicolas Puig, est un marqueur de la fête : au son suramplifié, saturé - c'est d'ailleurs, plus largement, un trait du paysage du Caire, fait-il observer à juste titre -, s'ajoutent une distorsion systématique et l'usage emphatique de l'écho.

5 «L'Avenue » (ash-shâr'i), l'avenue Mohamed-Ali percée au milieu du XIx $x^{e}$ siècle dans le tissu ancien pour relier la citadelle au nouveau centre-ville, a été pendant des décennies le creuset où les musiciens de fête et leur musique se sont formés. Qu'ils y résident ou qu'ils habitent à proximité, elle a été leur territoire, et celui des chanteuses et des danseuses (les almées). Sous ses arcades on trouvait les nombreuses échoppes des fabricants et des marchands d'instruments peu à peu remplacées par des magasins et des ateliers de meubles. Dans les cafés de l'Avenue, lieux de regroupement corporatif et d'embauche, où les gens de radio, puis de télévision, avaient également leurs habitudes, 
les musiciens étaient recrutés pour une soirée, le temps d'un concert, d'une fête ou davantage. Il fallait "avoir sa chaise » dans un de ces "cafés de musiciens » (il n'en reste que deux), et la fréquentation de tel ou tel établissement plus ou moins prestigieux était pour un artiste l'indice indiscutable de sa cote. À l'époque où se développait l'industrie du disque et où se déroulaient d'intenses débats, dont le Congrès de musique arabe du Caire (1922) fut le théâtre, c'est là que fut inventée la « chanson de variété longue» qui eut un impact décisif sur l'évolution de la chanson arabe, régénérée notamment par la chanteuse Umm Kalthum. Même si, d'ordinaire, les classes aisées méprisaient les musiciens, le prestige de l'Avenue était grand, dans le monde musical au moins. Pour un nouveau venu dans la profession, elle était la place à conquérir.

6 L'avenue Mohamed-Ali a été longtemps identifiée à l'almée, figure fantasmatique, à la fois séductrice, consolatrice et vénale, qui fascina tant Gustave Flaubert. Danseuse et chanteuse dont l'existence est attestée dès le XVIII siècle en Égypte, elle est un personnage de roman, par exemple chez Naguib Mahfouz, et de film jusqu'aux années 1970. Le terme "almée » est l'adaptation en français du mot arabe 'âlima, qui signifie "savante». L'orientaliste Maxime Rodinson lui a consacré un article érudit dans L'Encyclopédie de l'islam. Pas de farah sans " almée ", rappelle Nicolas Puig, qui souligne «l'intrication des destins des musiciens et des danseuses ». Celle-ci « explique pourquoi désormais le terme 'awalîm [pluriel de 'alima] est employé dans certains contextes pour désigner, avec une nette connotation péjorative, les artistes des deux sexes, et non pas uniquement les femmes» (p. 75). Ce glissement terminologique correspond à une aggravation de la réprobation morale (à cause du spectre du sexe et de l'alcool) et de la stigmatisation sociale dont font l'objet les musiciens, féminisés en l'occurrence.

7 Se voulant les dépositaires d'un art dont ils revendiquent l'authenticité, affirmant leur amour du métier, ces artistes déplorent que, sous la pression de la commande, celui-ci se perde et que les instruments traditionnels - en premier lieu le luth, réhabilité par le renouveau de la musique arabe classique - soient détrônés par d'autres plus modernes et bruyants, des claviers électroniques principalement, ou même des batteries. Traités de mazikâtî, expression dévalorisante, et non de muzîkâr, "maître musicien", les artistes de rue se sont paupérisés. Ils sont marginalisés, suivant une évolution concomitante de l'Avenue et du public lui-même, méprisé pour sa pauvreté. Et ils se sont dispersés dans l'espace : « Il y a maintenant une avenue Mohamed-Ali dans tous les quartiers, dans toutes les villes [...], dans tous les villages », déclare le maître Ahmad Wahdan (p.132).

8 L'enquête de Nicolas Puig, qui s'inscrit dans la lignée d'Howard Becker, se réfère aux Mondes de l'art lorsqu'il décrit la division du travail et la coopération qui caractérisent le farah, et à Outsider, où Becker évoque le statut dévalorisé du musicien de danse à Chicago dans les années 1940. Au Caire, la profession est souvent inavouable, celui qui l'exerce la dissimule à ses voisins, voire à sa famille, et à ses collègues s'il occupe un second emploi jugé respectable. Les musiciens ont tendance pour leur part à considérer leur activité comme un travail alimentaire, aléatoire de surcroît. Ils évoquent leur précarité, les horaires épuisants, la course au cachet, la recherche d'un extra dans une boîte de nuit ou un hôtel, les embrouilles du nabatshi qu'il faut déjouer... et la menace que représente l'intervention croissante des DJ. Le champ musical n'est plus qu'un sûq, disent-ils - un souk, un marché. Et il semble bien que, dans le farah, le rapport à l'argent soit de plus en plus prégnant et ostentatoire, d'où l'importance croissante du 
nabatshi. L'auteur suggère qu'une telle tendance est liée à la politique de libéralisation économique initiée au milieu des années 1970 par Anouar el-Sadate.

Si pour l'essentiel il ne ressortit pas à l'ethnomusicologie stricto sensu, l'ouvrage de Nicolas Puig appréhende l'objet musical dans sa spécificité, en particulier à travers les quatre récits très personnels qu'il a recueillis. Ceux-ci détaillent les situations observées par l'ethnologue, qui ne se limitent pas aux fêtes de rue, et mettent en lumière l'amour de la musique exprimé par leurs auteurs, revenant sur leur découverte (au moins pour trois d'entre eux, le quatrième la présentant comme une simple occasion qui s'offre à lui de gagner un peu d'argent) : "J'ai commencé à taper en rythme sur les tables, raconte Mahmoud. Sur n'importe quoi. Les percussions m'ont plu. Et je me suis mis à taper sans arrêt des rythmes sur les tables, les chaises [...]» (p. 159). Ces artistes décrivent l'éveil de leur pratique musicale, les obstacles qu'ils ont dû vaincre, leur apprentissage et l'entrée dans la profession. Ils expliquent comment ils inventent, à chaud, des solutions aux problèmes mélodiques, rythmiques, modaux qui surgissent lorsqu'ils jouent avec d'autres instrumentistes. Mahmoud décrit en outre la façon dont un percussionniste doit suivre la danseuse. Une leçon. Comparant cet accompagnement, plus rythmique et varié, à celui d'un chanteur, il conclut, après deux années passées auprès de la célèbre Fifi Abdou dans le night-club d'un hôtel de luxe, loin du farah, avant de revenir aux mariages et aux boîtes de nuit: «Le bon tabbâl ne doit jamais quitter la danseuse des yeux. Quoi qu'elle fasse, il faut la suivre, même si c'est faux, car cela la met à l'aise en tant que danseuse » (p. 165).

L'autre versant du travail de Nicolas Puig, on l'aura compris, a pour objet l'espace du Caire. Avec une grande finesse, son enquête d'ethnologie urbaine rend intelligibles les complexes topographies en mouvement que dessinent les musiciens de noces autour de ce qu'il appelle « la rue festive » $:$ la rue du farah.

\section{NOTES}

1. Deux regrets : l'absence d'un glossaire qui systématise et explicite les termes musicaux (par exemple la différence entre les deux sortes de tambours à cadre que sont le duff et le riq) et celle d'un plan, même sommaire, du Caire.

\section{AUTEURS}

\section{JEAN-CHARLES DEPAULE}

jean-charles.depaule898@orange.fr 